\title{
Cake kidney drained though a single ureter in a patient with recurring urinary tract infection
}

\author{
Bianca Aki Ishiy Ozima ${ }^{1}$, José Germano Ferraz de Arruda ${ }^{2}$, Marcio Gatti $^{2}$, \\ Germano José Ferraz de Arruda ${ }^{2}$, Luís Cesar Fava Spessoto ${ }^{2}$, Fernando Nestor Fácio Jr ${ }^{2}$ \\ ${ }^{1}$ General Surgery, Hospital de Base/FUNFARME, São José do Rio Preto, SP, Brazil; ${ }^{2}$ Department of Urology, São José do Rio Preto School of \\ Medicine (FAMERP/FUNFARME), São José do Rio Preto, SP, Brazil \\ Correspondence to: Fernando Nestor Fácio Jr. São José do Rio Preto School of Medicine, Ave. Brig. Faria Lima 5416, São José do Rio Preto, SP 15090- \\ 000, Brazil. Email: fnfacio@yahoo.com.br.
}

\begin{abstract}
Cake kidney is a rare abnormality of the urinary tract. It is generally asymptomatic but can lead to renal and nonspecific systemic manifestations. This report describes a case of cake kidney drained through a single ureter diagnosed in a female patient with recurring urinary infection.
\end{abstract}

Keywords: Kidney; abnormalities; ureter

Submitted Jul 04, 2019. Accepted for publication Sep 29, 2019.

doi: $10.21037 /$ tau.2019.10.14

View this article at: http://dx.doi.org/10.21037/tau.2019.10.14

\section{Introduction}

Cake kidney is a rare congenital abnormality of the genitourinary tract commonly drained by two ureters. There are only six cases of cake kidney drained though single ureter in the literature (1). The early diagnosis and the identification of possible complications of this anomaly are essential to prevent permanent renal damage (2).

In this work, the authors present a case of cake kidney drained though a single ureter in a patient with recurring urinary tract infection.

\section{Case presentation}

A 49-year-old female patient with a history of recurring urinary tract infection for several years treated with different antibiotics, including prophylactic treatment with nitrofurantoin, was sent to the urology clinic of a tertiary level teaching school for an ultrasound exam of the pelvic left kidney.

The laboratory exams revealed urinary tract infection with E. coli sensitive to all antibiotics and preserved kidney function (creatinine: $0.8 \mathrm{mg} / \mathrm{dL}$ ). Voiding urethrocystography revealed no abnormalities. Dynamic renal scintigraphy revealed the functional exclusion of the right kidney, pelvic left kidney with preserved renal function and patent left excretory duct. Computed tomography of the complete abdomen with contrast revealed fused kidneys in topography from the midline below the bifurcation of the aorta, smooth contours of the "cake" kidney, medium thickness of the conserved parenchyma and a single urethra (Figure $1 A, B$ ).

The patient has not presented any further episodes of urinary tract infection and is asymptomatic. Due to the concomitance with other abnormalities, the patient is currently in outpatient follow-up.

\section{Discussion}

Cake kidney can be diagnosed at any age and, like other renal fusion abnormalities, is more common in men, with a proportion of 2-3:1 (3). Patients are generally asymptomatic, but can present with fever, low abdominal pain as well as a predisposition toward to the formation of calculi and recurring urinary tract infection due to the anomaly of the collector system and short ureters, which are prone to stasis and obstruction (4).

The diagnosis is always incidental (4). The anomaly is generally detected first during exams such as ultrasound or excretory urography, but computed tomography enables 

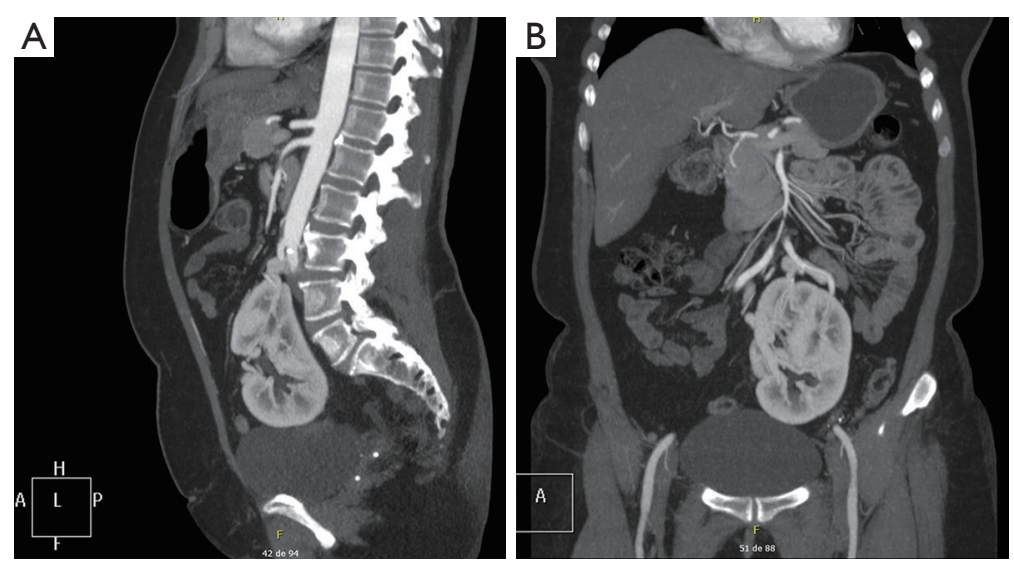

Figure 1 Abdominal computed tomography showing cake kidney. (A) sagittal cut; (B) coronal cut.

a precise diagnosis. Moreover, computed tomography urography with multi-detectors may be employed, with enables a better evaluation of the congenital anomalies of the urinary tract and ureters (5).

This abnormality is not necessarily associated with kidney dysfunction or a prognosis of the progressive deterioration of kidney function (6). However, the patient should be investigated with regard to other possible concomitant anomalies, such as tetralogy of Fallot, vaginal agenesis, spina bifida, abnormal testicular descent, sacral agenesis and anal abnormalities. Moreover, long-term follow-up of kidney function is necessary for the early detection of complications, such as obstruction, calculosis, infection, hematuria and uremia, thereby reducing the occurrence of associated morbidities (7).

\section{Acknowledgments}

None.

\section{Footnote}

Conflicts of Interest: The authors have no conflicts of interest to declare.

Ethical Statement: The authors are accountable for all aspects of the work in ensuring that questions related to the accuracy or integrity of any part of the work are appropriately investigated and resolved. Informed consent was obtained from the patient in clinical consents to use radiologic imaging and to publication of this case report.

\section{References}

1. Calado AA, Macedo A Jr, Srougi M. Cake kidney drained by single ureter. Int Braz J Urol 2004;30:321-2.

2. Türkvatan A, Demir D, Olçer T, et al. Cake kidney: MDCT urography for diagnosis. Clin Imaging 2006;30:420-2.

3. Kaufman MH, Findlater GS. An unusual case of complete renal fusion giving rise to a 'cake' or 'lump' kidney. J Anat 2001;198:501-4.

4. Tiwari AK, Choudhary AK, Khowal H, et al. Pancake kidney: A rare developmental anomaly. Can Urol Assoc J 2014;8:E451-2.

5. Türkvatan A, Olçer T, Cumhur T. Multidetector CT urography of renal fusion anomalies. Diagn Interv Radiol 2009;15:127-34.

6. Glenn JF. Fused pelvic kidney. J Urol 1958;80:7-9.

7. Martínez-Lázaro R, Cortés-Blanco A. Cake kidney drained by single ureter: MAG3 renogram for diagnosis and function follow-up. Nephrol Dial Transplant 2000;15:1700-1.

Cite this article as: Ozima BAI, de Arruda JGF, Gatti M, de Arruda GJF, Spessoto LCF, Fácio FN Jr. Cake kidney drained though a single ureter in a patient with recurring urinary tract infection. Transl Androl Urol 2019;8(6):762-763. doi: 10.21037/ tau.2019.10.14 